\title{
Generalized synchronization in complex dynamical networks via adaptive couplings
}

Liu, Hui; Chen, Juan; Lu, Jun-an; Cao, Ming

Published in:

Physica A: Statistical Mechanics and its Applications

DOI:

10.1016/j.physa.2009.12.035

IMPORTANT NOTE: You are advised to consult the publisher's version (publisher's PDF) if you wish to cite fro it. Please check the document version below.

Document Version

Publisher's PDF, also known as Version of record

Publication date:

2010

Link to publication in University of Groningen/UMCG research database

Citation for published version (APA):

Liu, H., Chen, J., Lu, J., \& Cao, M. (2010). Generalized synchronization in complex dynamical networks v adaptive couplings. Physica A: Statistical Mechanics and its Applications, 389(8), 1759-1770. https://doi.org/10.1016/j.physa.2009.12.035

\section{Copyright}

Other than for strictly personal use, it is not permitted to download or to forward/distribute the text or part of it without the consent of the author(s) and/or copyright holder(s), unless the work is under an open content license (like Creative Commons).

The publication may also be distributed here under the terms of Article $25 \mathrm{fa}$ of the Dutch Copyright Act, indicated by the "Taverne" license More information can be found on the University of Groningen website: https://www.rug.nl/library/open-access/self-archiving-pure/taverneamendment.

Take-down policy

If you believe that this document breaches copyright please contact us providing details, and we will remove access to the work immediate and investigate your claim.

Downloaded from the University of Groningen/UMCG research database (Pure): http://www.rug.nl/research/portal. For technical reasons $t$ number of authors shown on this cover page is limited to 10 maximum. 


\title{
Generalized synchronization in complex dynamical networks via adaptive couplings
}

\author{
Hui Liu ${ }^{\mathrm{a}, \mathrm{b}, *}$, Juan Chen ${ }^{\mathrm{a}}$, Jun-an Lu ${ }^{\mathrm{a}}$, Ming Cao ${ }^{\mathrm{b}}$ \\ a School of Mathematics and Statistics, Wuhan University, Wuhan 430072, China \\ ${ }^{\mathrm{b}}$ Faculty of Mathematics and Natural Sciences, ITM, University of Groningen, Groningen, 9747 AG, The Netherlands
}

\section{A R T I C L E I N F O}

\section{Article history:}

Received 30 July 2009

Received in revised form 2 November 2009

Available online 29 December 2009

\section{Keywords:}

Complex dynamical network

Generalized synchronization

Adaptive coupling strength

Heterogeneity

\begin{abstract}
A B S T R A C T
This paper investigates generalized synchronization of three typical classes of complex dynamical networks: scale-free networks, small-world networks, and interpolating networks. The proposed synchronization strategy is to adjust adaptively a node's coupling strength based on the node's local generalized synchronization information. By taking the auxiliary-system approach and using the Lyapunov function method, we prove that for any given initial coupling strengths, the generalized synchronization can take place in complex networks consisting of nonidentical dynamical systems. It is demonstrated that the coupling strengths are affected by topologies of the networks. Furthermore, it is found that there are hierarchical features in the processes of generalized synchronization in scale-free networks because of their highly heterogeneous distributions of connection degree. Finally, we discuss in detail how a network's degree of heterogeneity affects its generalization synchronization behavior.
\end{abstract}

(C) 2010 Elsevier B.V. All rights reserved.

\section{Introduction}

In the past few years, one of the central themes in the study of complex networks consisting of coupled nonlinear dynamical systems has been to analyze the collective behaviors in small-world networks, scale-free networks and random networks [1-4]. Criteria are derived for complete synchronization in dynamical networks for which the interaction between network topologies and node dynamics is crucial [5-7]. Most of the existing literature on complete synchronization usually assumes that the dynamics of all the nodes in a network are exactly identical. This assumption, however, cannot be satisfied in many real situations because of parameter variation, unmodeled dynamics and random perturbations in real complex systems. Furthermore, it has been found that generalized synchronization, which is weaker than complete synchronization, plays an important role in engineering networks [8-10], biological systems [11], social activities, and many other fields. Therefore, it is of great interest to investigate generalized synchronization in networks consisting of nodes with nonidentical dynamics.

Generally speaking, the methods to achieve generalized synchronization can be divided into two classes. One approach is to design control laws to force coupled systems to satisfy a prescribed functional relation [12,13,11]. But this approach has the disadvantage that the designed controllers are usually quite complicated and thus difficult to be implemented in real applications. The other approach is the auxiliary-system approach, proposed by Abarbanel et al. [10], which was originally utilized to study chaotic behaviors and has been generalized to investigate problems in complex networks [14-16]. Some recent work $[14,15]$ has studied generalized synchronization in complex networks with the same coupling strength

\footnotetext{
* Corresponding author at: Faculty of Mathematics and Natural Sciences, ITM, University of Groningen, Groningen, 9747 AG, The Netherlands. Tel.: +31 513634770 .

E-mail addresses: hui.liu@rug.nl (H. Liu), juanchen1220@yahoo.com.cn (J. Chen), jalu@whu.edu.cn (J.-a. Lu), m.cao@rug.nl (M. Cao).
} 
among all nodes, and shown how different coupling strategies can affect the development of generalized synchronization. Motivated by these work, researchers have started to look at the more challenging scenario where the strengths of couplings among nodes in a network can be adaptively adjusted. Such scenario arise naturally in engineering, biological and social networks, where the coupling strengths of the interacting components are not static, but change adaptively with time [17-20]. The research on adaptive couplings in complex dynamical networks provides a new method to investigate the synchronization of large-scale complex networks. It has been pointed out in Ref. [21] that adaptive coupling strategies can enhance significantly the synchronizability of networks. But the existing work on this topic all focuses on the complete synchronization of networks that consists of identical dynamical nodes. In this paper, we use the idea of adaptive coupling to study generalized synchronization of complex dynamical networks. To be more specific, based on the construction of a Lyapunov function, we propose a novel control strategy to achieve generalized synchronization of complex dynamical networks consisting of nonidentical nodes by adaptively adjusting the coupling strengths according to the local generalized synchronization information.

We investigate the process of generalized synchronization (GS) in small-world networks and scale-free networks. By comparison, we find that the generalized synchronization processes in scale-free networks and small-world networks are quite different due to their distinct topological properties. In scale-free networks, generalized synchronization generally starts from the nodes with large degrees that are determined by the heterogeneity in the degree distribution. On the other hand, in small-world networks, there is no apparent spreading-out process of generalized synchronization because of the homogeneous degree distribution. And we find that scale-free networks need more time to achieve generalized synchronization than small-world networks of similar sizes and average node degrees. It is shown numerically that when generalized synchronization is achieved, the coupling strengths, which have been in stable states, become negatively correlated with node degrees.

This paper is organized as follows. In the next section, the model description and preliminaries are presented. In Section 3, using the auxiliary-system method and adaptive coupling strength scheme, a sufficient condition for generalized synchronization is derived. In Section 4, numerical simulations are carried out in complex networks in which each node is governed by the classical Lorenz system dynamics. Finally, discussion and concluding remarks are given in Sections 5 and 6 , respectively.

\section{Complex network model with adaptive couplings and preliminaries}

We consider a complex dynamical network consisting of $N$ nonidentical coupled nodes, each of which can be modeled by an $n$-dimensional dynamical system $\dot{x}_{i}(t)=f_{i}\left(x_{i}(t)\right), i=1,2, \ldots, N$. The network with adaptive coupling strengths can be written in the following form:

$$
\dot{x}_{i}(t)=f_{i}\left(x_{i}(t)\right)-c_{i}(t) \sum_{j=1}^{N} a_{i j} \Gamma\left(x_{i}(t)-x_{j}(t)\right), \quad i=1,2, \ldots, N,
$$

where $x_{i}(t)=\left(x_{i 1}(t), x_{i 2}(t), \ldots, x_{i n}(t)\right)^{T} \in R^{n}$ is the state of node $i, f_{i}: R^{n} \rightarrow R^{n}$ is a continuous vector function, and $c_{i}(t)>0$ is a time-varying coupling strength that will be designed according to node $i$ 's information about its neighborhood. The concrete adaptive control laws of $c_{i}(t)$ for $i=1,2, \ldots, N$, will be designed in the next section, in order to realize GS of the complex dynamical network (1). $\Gamma \in R^{n \times n}$ is an inner coupling matrix and is a diagonal matrix with positive diagonal elements. $A=\left(a_{i j}\right)$ is the adjacency matrix of the network; to be more specific, if there is a connection between node $i$ and node $j, a_{i j}=a_{j i}=1$, and otherwise $a_{i j}=a_{j i}=0$, and $a_{i i}=0$. In what follows, we assume that the network is connected.

In order to state our main result, we make the following assumption:

Assumption 1 (Global Lipschitz Condition). Suppose that there exist nonnegative constants $L_{i}$ for all $i=1,2, \ldots, N$, such that for any time-varying vectors $x(t), y(t) \in R^{n}$

$$
\left\|f_{i}(x(t))-f_{i}(y(t))\right\| \leq L_{i}\|x(t)-y(t)\|, \quad i=1,2, \ldots, N
$$

where $\|\cdot\|$ denotes the 2 -norm throughout the paper.

\section{The auxiliary-system approach for GS on complex dynamical networks via adaptive couplings}

The auxiliary-system approach has been widely used to detect the generalized synchronization in coupled chaotic systems $[10,14-16]$. In this section, in order to derive the condition for generalized synchronization, we apply the auxiliary-system method to complex networks with adaptive coupling strengths. However, it needs to be pointed out that this approach is not able to decide what functional relations exist between nodes when they achieve generalized synchronization. If one only wants to show the generalized synchronization in a network rather than the exact functional relations, this approach is an appropriate choice. Consider a complex dynamical network that consists of coupled systems via adaptive couplings. For any given node in this network, the coupling from neighboring nodes can be taken to be external 
driving forces as described in Refs. [14,15]. Therefore, we make a replica of each system in Eq. (1), resulting in the following network:

$$
\dot{x}_{i}^{\prime}(t)=f_{i}\left(x_{i}^{\prime}(t)\right)-c_{i}(t) \sum_{j=1}^{N} a_{i j} \Gamma\left(x_{i}^{\prime}(t)-x_{j}(t)\right), \quad i=1,2, \ldots, N .
$$

According to the auxiliary-system approach [10], the dynamical network (1) achieves GS, if $\lim _{t \rightarrow \infty}\left\|x_{i}(t)-x_{i}^{\prime}(t)\right\|=0$ for any initial conditions $x_{i}(0) \neq x_{i}^{\prime}(0)$, where $i=1,2, \ldots, N$.

Define $e_{i}(t)=x_{i}^{\prime}(t)-x_{i}(t)$. Subtracting (1) from (3), we have

$$
\dot{e}_{i}(t)=f_{i}\left(x_{i}^{\prime}(t)\right)-f_{i}\left(x_{i}(t)\right)-c_{i}(t) \sum_{j=1}^{N} a_{i j} \Gamma e_{i}(t), \quad i=1,2, \ldots, N .
$$

Theorem 1. Suppose Assumption 1 holds. Using the adaptive controller

$$
\dot{c}_{i}(t)=\gamma_{i} \sum_{j=1}^{N} a_{i j} e_{i}(t)^{T} \Gamma e_{i}(t), \quad i=1,2, \ldots, N
$$

where $\gamma_{i}$ are positive constants, the dynamical networks (1) and (3) can be synchronized as $t \rightarrow \infty$, i.e. the complex network (1) achieves generalized synchronization.

Proof. We choose to use the following Lyapunov function candidate

$$
V=\frac{1}{2} \sum_{i=1}^{N}\left(x_{i}^{\prime}(t)-x_{i}(t)\right)^{T}\left(x_{i}^{\prime}(t)-x_{i}(t)\right)+\frac{1}{2} \sum_{i=1}^{N} \frac{1}{\gamma_{i}}\left(c_{i}(t)-c^{*}\right)^{2} .
$$

The time derivative of $V(t)$ along the solution of Eq. (4) is

$$
\begin{aligned}
\dot{V} & =\sum_{i=1}^{N} e_{i}(t)^{T} \dot{e}_{i}(t)+\sum_{i=1}^{N} \frac{1}{\gamma_{i}}\left(c_{i}(t)-c^{*}\right) \dot{c}_{i}(t) \\
& =\sum_{i=1}^{N} e_{i}(t)^{T}\left\{f_{i}\left(x_{i}^{\prime}(t)\right)-f_{i}\left(x_{i}(t)\right)-c_{i}(t) \sum_{j=1}^{N} a_{i j} \Gamma e_{i}(t)\right\}+\sum_{i=1}^{N} \frac{1}{\gamma_{i}}\left(c_{i}(t)-c^{*}\right) \dot{c}_{i}(t) \\
& =\sum_{i=1}^{N} e_{i}(t)^{T}\left\{f_{i}\left(x_{i}^{\prime}(t)\right)-f_{i}\left(x_{i}(t)\right)\right\}-\sum_{i=1}^{N} e_{i}(t)^{T} c_{i}(t) \sum_{j=1}^{N} a_{i j} \Gamma e_{i}(t)+\sum_{i=1}^{N} \frac{1}{\gamma_{i}} c_{i}(t) \dot{c}_{i}(t)-\sum_{i=1}^{N} \frac{1}{\gamma_{i}} c^{*} \dot{c}_{i}(t) \\
& \leq \sum_{i=1}^{N} L_{i} e_{i}(t)^{T} e_{i}(t)-\sum_{i=1}^{N} e_{i}(t)^{T} c_{i}(t) \sum_{j=1}^{N} a_{i j} \Gamma e_{i}(t)+\sum_{i=1}^{N} \frac{1}{\gamma_{i}} c_{i}(t) \dot{c}_{i}(t)-\sum_{i=1}^{N} \frac{1}{\gamma_{i}} c^{*} \dot{c}_{i}(t) .
\end{aligned}
$$

Denote $L=\max \left\{L_{i} \mid i=1,2, \ldots, N\right\}$. In view of Eq. (5), one obtains

$$
\dot{V} \leq L \sum_{i=1}^{N} e_{i}(t)^{T} e_{i}(t)-c^{*} \sum_{i=1}^{N} \sum_{j=1}^{N} a_{i j} e_{i}(t)^{T} \Gamma e_{i}(t)=L \sum_{i=1}^{N} e_{i}(t)^{T} e_{i}(t)-c^{*} \sum_{i=1}^{N} k_{i} e_{i}(t)^{T} \Gamma e_{i}(t),
$$

where $k_{i}=\sum_{j=1}^{N} a_{i j}$ is the degree of node $i$. Let $e(t)=\left(e_{1}(t)^{T}, e_{2}(t)^{T}, \ldots, e_{N}(t)^{T}\right)^{T} \in R^{n N}$ and $P=D \otimes \Gamma$, where $D=\operatorname{diag}\left\{k_{1}, k_{2}, \ldots, k_{N}\right\}$ is an $N \times N$ diagonal matrix, then we have

$$
\dot{V} \leq L e(t)^{T} e(t)-c^{*} e(t)^{T} P e(t) \leq e(t)^{T}\left(L-c^{*} \lambda_{\min }\left(\frac{P+P^{T}}{2}\right)\right) e(t) .
$$

Since the matrix $D$ is positive definite, and $P=D \otimes \Gamma$ is also positive definite, one obtains that $\lambda_{\min }\left(\frac{P+P^{T}}{2}\right)>0$. Taking a sufficiently large $c^{*}$, one has

$$
\dot{V} \leq-e(t)^{T} e(t)
$$

we have $e_{i}(t) \rightarrow 0$ as $t \rightarrow \infty$, i.e. $\left|x_{i}(t)-x_{i}^{\prime}(t)\right| \rightarrow 0$ as $t \rightarrow \infty$, which indicates that the complex dynamical network (1) achieves generalized synchronization.

Remark 1. The positive constants $\gamma_{i}$ can determine the synchronization or convergence speed. The convergence speed becomes faster for larger $\gamma_{i}$. 
Remark 2. Let $k_{i}=\sum_{j=1}^{N} a_{i j}$, where $k_{i}$ is the node degree of node $i$. Then the adaptive controllers in Eq. (5) can be rewritten as

$$
\dot{c}_{i}(t)=\gamma_{i} k_{i} e_{i}(t)^{T} \Gamma e_{i}(t), \quad i=1,2, \ldots, N .
$$

From the equation above, one can see that the acceleration of $c_{i}(t)$ is determined by the node' degree $k_{i}$ and its local generalized synchronization information $e_{i}(t)^{T} \Gamma e_{i}(t)$. It is different than the complete synchronization results in papers $[20,21]$, where the update of $c_{i}(t)$ also involves its neighboring nodes' errors $\left\{e_{j}(t) \mid j \in \mathscr{N}_{i}\right\}$, where $\mathscr{N}_{i}$ denotes all the neighbors of node $i$, i.e., $j \in \mathscr{N}_{i}$, if and only if $a_{i j}=a_{j i} \neq 0$ and $j \neq i$.

Remark 3. The result of Theorem 1 can be further extended to the case of weighted complex dynamical networks. Previously, we take $A=\left(a_{i j}\right)_{N \times N}$ as the adjacency matrix of the network. If $a_{i j}$ are also used to denote the weights $w_{i j}$ between nodes $i$ and $j$ for a weighted network, then one can check that Theorem 1 holds as well. Define the strength of node $i$ to be $s_{i}=\sum_{j=1}^{N} a_{i j}$, according to the definition in Ref. [22] for a weighted network, we choose to use the adaptive controllers

$$
\dot{c}_{i}(t)=\gamma_{i} s_{i} e_{i}(t)^{T} \Gamma e_{i}(t), \quad i=1,2, \ldots, N,
$$

where $\gamma_{i}$ are positive constants. Then the weighted complex dynamical network (1) achieves generalized synchronization as $t \rightarrow \infty$.

\section{Numerical simulations}

In this section, we investigate generalized synchronization for coupled nonidentical chaotic systems in both the scalefree and the small-world networks via adaptive couplings. Here we consider the scale-free networks which are generated by the Barabási-Albert (BA) preferential attachment algorithm [2]. Starting with $m_{0}$ fully connected nodes, at each time step a new node is added and connected to $m$ existing nodes in the network with the probability $\prod_{i}=k_{i} / \sum_{j} k_{j}$, which is a linear preferential attachment strategy. The resulting scale-free networks have a power-law degree distribution $p(k) \sim k^{-\delta}$ with $\delta=3$. In this paper, we consider small-world networks that are generated by the Newman-Watts (NW) algorithm [23]. The model is constructed on a lattice with $N$ nodes, each connected clockwise to its $K$ nearest neighbors by undirected edges. The average degree of this regular lattice is $2 K$. Then randomness is introduced by independently adding connections with probability $p$ between any pair of disconnected nodes. The resulting small-world networks are highly clustered with short average distances between nodes. We set $K=2$ in the following simulations.

In the simulations, we set the number of the nodes $N$ to be 800 . We take the nodes' dynamics to be the three-dimensional classical Lorenz systems, described by

$$
\dot{x}_{i}=f_{i}\left(x_{i}\right)=\left[10\left(x_{i 1}-x_{i 2}\right), \alpha_{i} x_{i 1}-x_{i 2}-x_{i 1} x_{i 3}, x_{i 1} x_{i 2}-(8 / 3) x_{i 3}\right]^{T} .
$$

In our study, the Lorenz oscillators are intentionally chosen to be different by the uniform randomization of $\alpha_{i}$ in the interval $[28.0,30.0]$. We choose the initial values of the coupling strengths randomly from the interval $(0,0.1)$, and set the constants $\gamma_{i}$ in controllers (5) to be 0.2 .

To describe the process of the generalized synchronization in network (2), we define the following error signals at each time step

$$
E_{i}(t)=\left\|x_{i}^{\prime}(t)-x_{i}(t)\right\|_{2}, \quad i=1,2, \ldots, N .
$$

We label the nodes according to their decreasing degree ordering $d(1) \geq d(2) \geq d(3) \cdots \geq d(N)$. Now we analyze the development of the generalized synchronization from the following different aspects: the nodes' degrees, the properties of the networks' topological structures, coupling strengths, and hierarchical properties.

\subsection{Nodes' degrees}

Numerical results illustrated in Figs. 1 and 2 show the process of the global generalized synchronization. Fig. 1(a) shows the synchronization process of a scale-free network with $m_{0}=5, m=3$, which has been introduced in the previous section. Fig. 1(b) shows the evolution processes of $E_{i}(t), i=2,202,402$ for $t \in[0,1.0]$. Fig. 2(a) presents the synchronization procedure of a small-world network generated by the NW model, with the link probability $p=0.0025$, and Fig. 2 (b) presents the evolution processes of $E_{i}(t), i=2,202,402$ for $t \in[0,1.0]$. The total edges in the BA network and the NW network are both $2388 \pm 2$.

One can easily see that the nodes do not achieve a common generalized synchronization state at the same time. It is obvious that there is a transition process between the non-generalized synchronization and the global generalized synchronization. Take Fig. 1(a) as an example. When $t=0$, none of the node is in the generalized synchronization state at all. As time evolves to $0.2<t<0.6$, some nodes achieve generalized synchronization; however the others do not. When time $t>0.8$, most of the nodes are in generalized synchronization states. Similar phenomena can also be observed in the small-world network. 

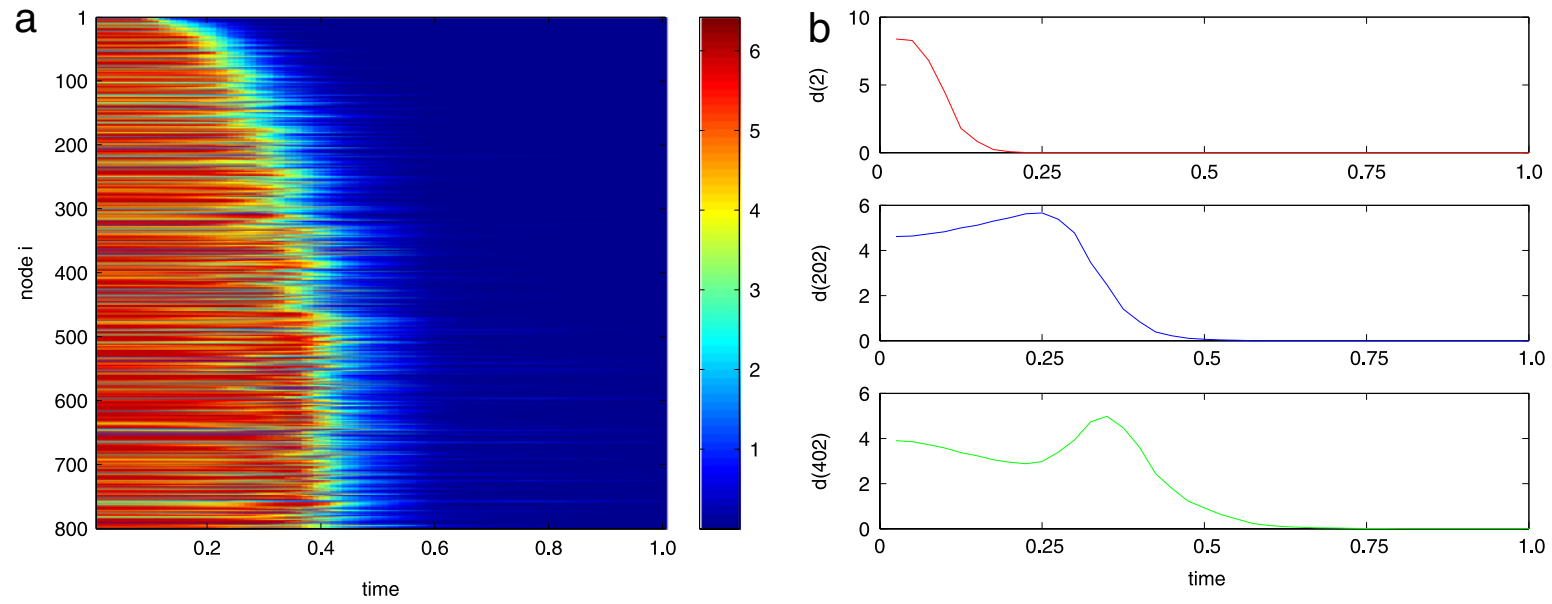

Fig. 1. Visualization of the process to global generalized synchronization via adaptive couplings in a scale-free (BA) network. The number of nodes is $N=800$ and the number of edges is $2388 \pm 2$. Subfigure (a) is a color map of $E_{i}(t)$ in the two-dimensional parameter space $(t, i)$, characterizing the development of the generalized synchronization. Subfigure (b) describes the evolution process of $E_{i}(t)$ for different nodes $i=2,202,402$. (For interpretation of the references to colour in this figure legend, the reader is referred to the web version of this article.)
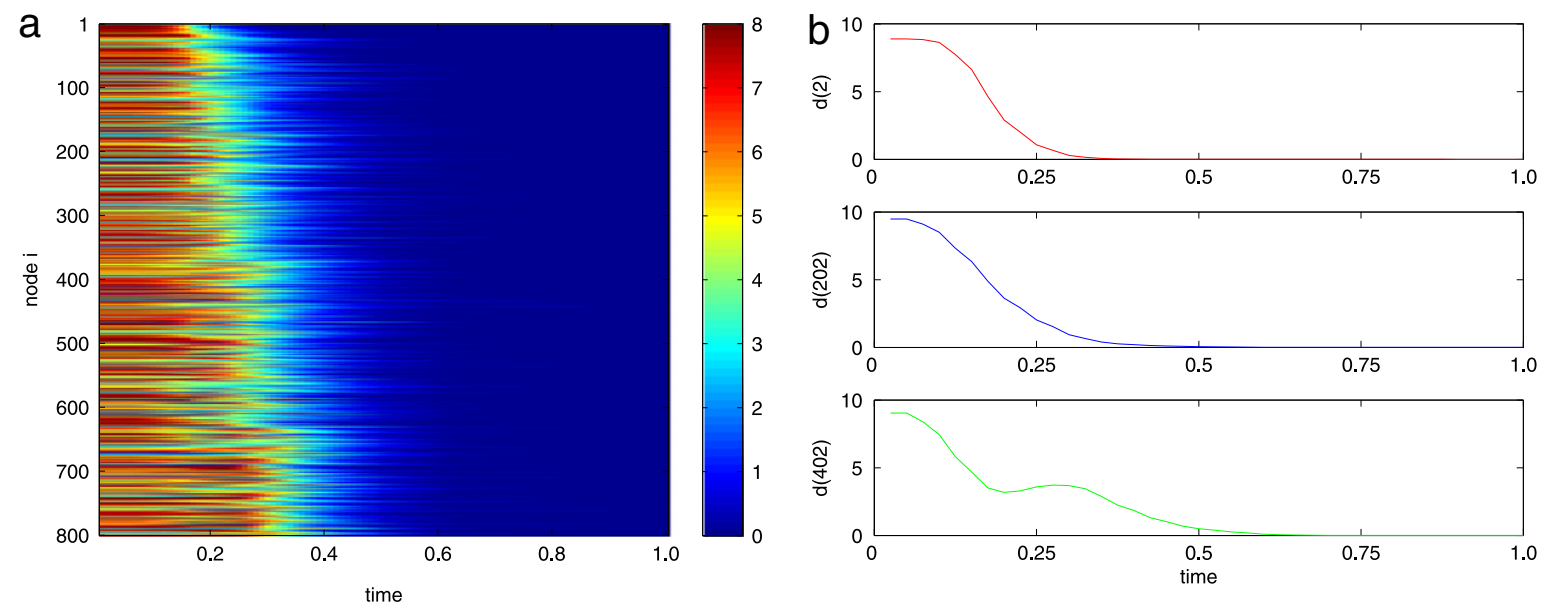

Fig. 2. Visualization of the process to global generalized synchronization via adaptive couplings in a small-world (NW) network. The number of nodes is $N=800$, and the number of edges is $2388 \pm 2$. Subfigure (a) is a color map of $E_{i}(t)$ in the two-dimensional parameter space $(t, i)$, characterizing the development of the generalized synchronization. Subfigure (b) describes the evolution process of $E_{i}(t)$ for different nodes $i=2,202,402$. (For interpretation of the references to colour in this figure legend, the reader is referred to the web version of this article.)

As shown in Fig. 1(a), as the system evolves, the generalized synchronization starts from the nodes with the largest degree and then spreads to the rest of the network. It can be derived from Eq. (4), in which the linearization results is

$$
\dot{e}_{i}=\left(D f_{i}\left(x_{i}\right)-c_{i}(t) \sum_{j=1}^{N} a_{i j} \Gamma\right) e_{i} .
$$

Here, $D f_{i}\left(x_{i}\right)$ denotes the Jacobian matrix of $f_{i}(x)$ at $x_{i}$. In Eq. (8), it is clear that $\sum_{j=1}^{N} a_{i j}$ is the degree of node $i$. Hence, the greater a node's degree is, the faster the error converges to zero, which is validated by Figs. 1(b) and 2(b). Furthermore, by comparing Fig. 1 with Fig. 2, it can be seen that the processes of generalized synchronization in the two kinds of networks, i.e. the scale-free network (BA) and the small-world network (NW), are quite different: the phase of the partial generalized synchronization for the scale-free network is much longer than that of the small-world network. For the scale-free network, the node with the largest degree achieves generalized synchronization at the time less than $t=0.2$, and the nodes with the smallest degree almost achieve generalized synchronization at time $t=0.9$. As for the small-world network, the node with the largest degree achieves generalized synchronization around $t=0.3$, and the nodes with the smallest degree achieve generalized synchronization around $t=0.6$. However, according to the color bars in the two figures, the conclusions are draw based on the assumption that the network achieve generalized synchronization roughly at the time when the errors $E_{i}(t)<0.5$. The two networks behave differently because they have different distributions of nodes' degrees. 

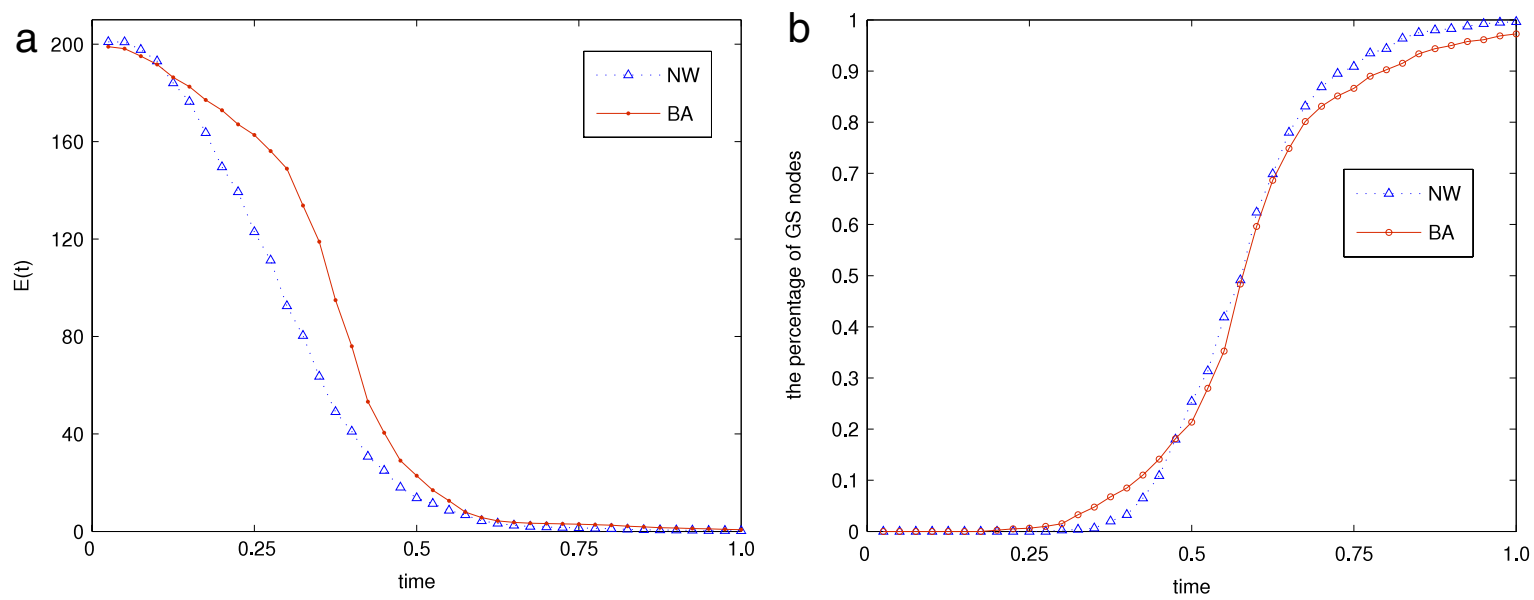

Fig. 3. A comparison between the BA network model and the NW network model. (a) shows the evolution of the error $E(t)$. It shows that the synchronization speed of the small-world network is faster than that of the scale-free network. (b) shows the percentage of the nodes which have achieved generalized synchronization in the adaptive evolution process. It indicates that at an early stage, the scale-free network is easier to be synchronized, but it is harder to do so compared to the small-world network later on.

\subsection{Properties of networks' topological structures}

In the previous section, we have shown that there is a transition process before all nodes achieve global generalized synchronization in both the scale-free network and the small-world network. In this section, we focus on how the topological structures in these two types of complex networks affect the generalized synchronization process. Here, we define $E(t)=$ $\sum_{i=1}^{N} E_{i}(t)$. Fig. 3(a) shows the evolution of $E(t)$ both for a scale-free network and a small-world network, while Fig. 3(b) shows the percentage of the synchronized nodes in the generalized synchronization process. In the computation, node $i$ is considered to be in the generalized synchronization state when $E_{i}(t) \leq \Delta_{\text {thr }}$ at time $t \in(0,1]$. Here, the threshold value $\Delta_{\text {thr }}$ is set to be 0.05 in the numerical simulation.

From Fig. 3, one can see that the scale-free network is easier to be in the generalized synchronization in the early stage, but harder in the later stage. This can be explained by the fact that the small-world network has comparative homogeneous nodes' degree distribution while the scale-free network has some nodes with extremely large degrees. In fact, in the beginning, the existence of these nodes with extraordinary degrees (much larger than the average value) accelerate the generalized synchronization speed in the scale-free network. But at a later stage, the speed to achieve generalized synchronization is dominated by the nodes with the smallest degrees. As for the scale-free network, the hub nodes achieve generalized synchronization quickly, but distant nodes are much more difficult to achieve generalized synchronization. So from the whole network point of view, it is easier for the small-world network (NW) to achieve global generalized synchronization than the scale-free network (BA) with the same numbers of nodes and edges.

Furthermore, we investigate how the probability $p$ affects the development of generalized synchronization for a smallworld network. We have carried out extensive numerical simulations for each network for the time interval $t \in[0,1.0]$ with step size $\delta t=0.025$. As shown in Fig. 4, we change the probability $p$ from 0.0015 to 0.02 , the percentages of nodes in generalized synchronization states are investigated. It can be seen from the figure that the NW small-world network with higher link probability $p$ is easier to be in generalized synchronization for all nodes.

\subsection{Coupling strengths}

Since we use adaptive coupling strengths to achieve generalized synchronization of complex dynamical networks, we need to investigate the evolution process of the coupling strengths. Fig. 5 illustrates the evolution of coupling strengths $c_{i}(t)$ for the BA network model, and Fig. 6 shows that for the NW network model. In Figs. 5(a) and 6(a), the evolutions of adaptively coupling strengths $c_{i}(t)$ for $i=6,106,206, \ldots, 706$ are shown. As shown in Figs. 5(b) and 6(b), every dot in both subfigures corresponds to the average value for a group of nearby 10 nodes, i.e. $\left.\frac{1}{10} \sum_{i=10(k-1)+1}^{10 k} c_{i}(t)\right|_{t=1.0}$, where $k=1,2, \ldots, 800 / 10$. The red solid line shows a linearly fitted curve for all dots in Fig. 5(b), as well as in Fig. 6(b). The fitting is done using CFTOOL in the Matlab toolbox. The slope for the fitting curve (red solid line) in Fig. 5 (b) is $0.3897 \times 10^{-2}$, and that for the fitting curve in Fig. 6(b) is $0.1953 \times 10^{-2}$.

From Figs. 5 and 6, one can see that almost every node has a different coupling strength, which is consistent with the real physical situations. In general, the node with larger degree needs smaller coupling strength. Therefore from Fig. 5(b), as the node's degree declines, the coupling strength changes with an upward trend. By examining Figs. 5(b) and 6(b), it is found that there is significant difference between the scale-free network and the small-world network: the slope of the rising solid line fitting for the coupling strengths in the scale-free network is twice as much as that in the small-world network. Because 


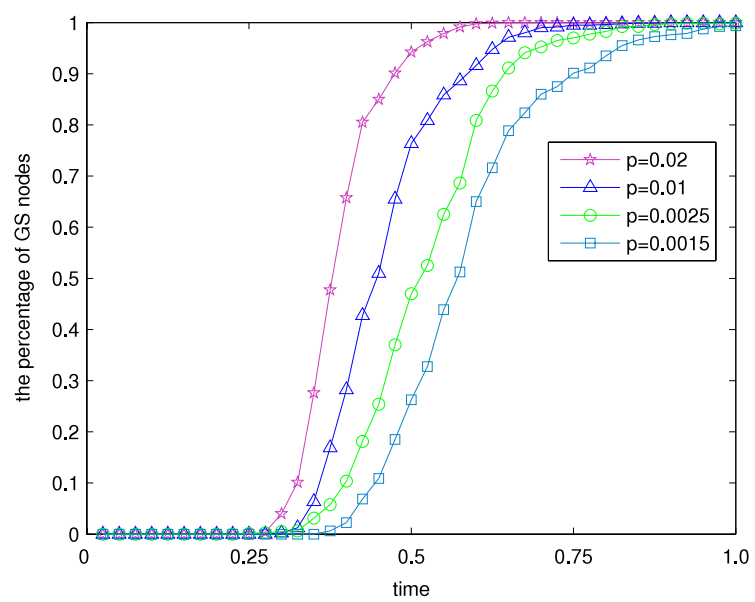

Fig. 4. A comparison between the NW networks generated by choosing different link probabilities $p$. It shows the percentages of the nodes that have achieved generalized synchronization in the adaptive evolution processes. It indicates that the NW small-world network with greater link probability $p$ is easier to be GS synchronized in the whole process.
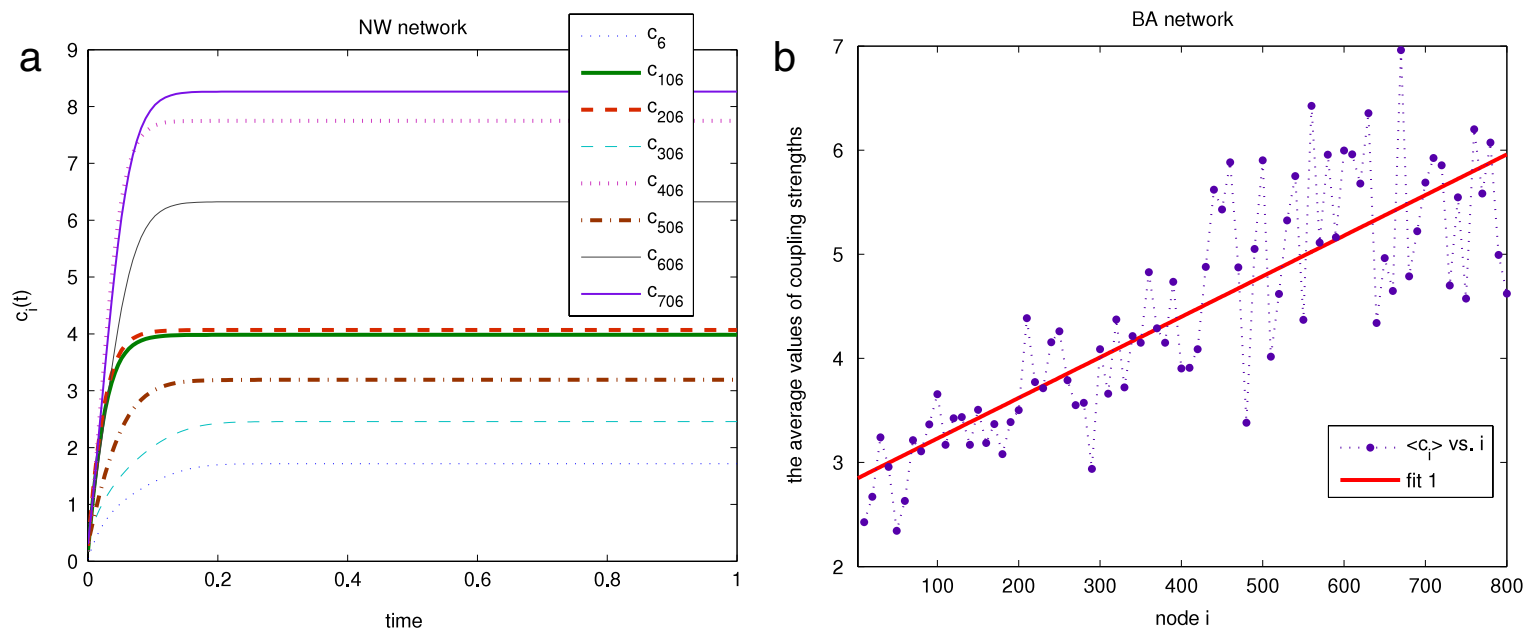

Fig. 5. The evolution of the coupling strengths $c_{i}(t)$ for the BA network model, with $m_{0}=5, m=3$, and $N=800$ nodes. Subfigure (a) is the process of the evolution of the coupling strengths $c_{i}(t)$ for $i=6,106,206, \ldots, 706$. Each dot plotted in subfigure (b) is the average value of the coupling strengths for 10 nodes $\left.\frac{1}{10} \sum_{i=10(k-1)+1}^{10 k} c_{i}(t)\right|_{t=1.0}$, where $k=1,2, \ldots, 800 / 10$. That is, we take every 10 nearby nodes in the ordering as a group. The $y$-coordinate represents the average value of the coupling strengths $\left.c_{i}(t)\right|_{t=1.0}$ in a group. (For interpretation of the references to colour in this figure legend, the reader is referred to the web version of this article.)

of the heterogeneous degree distribution, there is an obvious upward trend among the coupling strengths in the scale-free network. Due to the relative homogeneous degree distribution of the small-world, most nodes need approximately similar coupling strengths. However, it gradually shows a gentle upward trend for the case of the small-world network, since all nodes have been ranked from higher degrees to lower degrees $d(1) \geq d(2) \geq \cdots \geq d(N)$.

\subsection{Hierarchy in GS process}

In Section 4.1, we have shown that the generalized synchronization behaviors of the individual nodes (Lorenz oscillators) in scale-free network are quite nonuniform, since the nodes with large degrees have faster convergence rate. As a result, the generalized synchronization difference among them should be relatively small. As to their collective dynamics, a hierarchical structure may appear in the GS process. Inspired by how complete synchronization was studied in Ref. [24] for the purpose of clustering synchronization in scale-free networks, we define a dynamic correlation matrix $\&(t)$ for the pairwise GS errors $E_{i}(t), E_{j}(t)$ as

$$
\mathcal{E}_{i j}(t)= \begin{cases}1 & \text { if } E_{i} \leq \Delta_{t h r} \text { and } E_{j} \leq \Delta_{t h r} \\ 0 & \text { otherwise. }\end{cases}
$$

A pair of oscillators $i, j(i \neq j)$ are considered to both achieve generalized synchronization effectively at time $t$ when their correlation parameter $\mathcal{E}_{i j}(t)=1$. There is no unique choice of the threshold value of $\Delta_{t h r}$. With the smaller value of $\Delta_{t h r}$, 

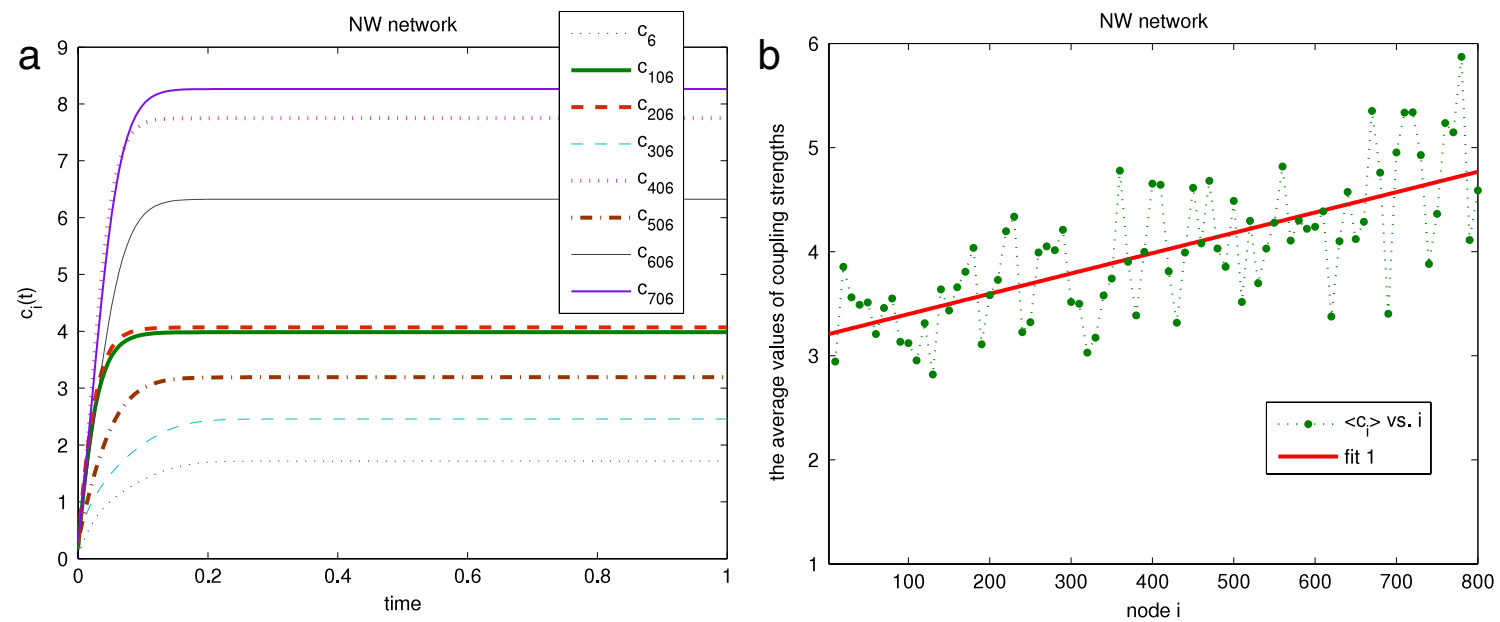

Fig. 6. The evolution of the coupling strengths $c_{i}(t)$ for the NW network model, with $p=0.002$, and $N=800$ nodes. Subfigure (a) is the process of the evolution of the coupling strengths $c_{i}(t)$ for $i=6,106,206, \ldots, 706$. Each dot plotted in subfigure (b) is the average value of the coupling strengths for 10 nodes $\left.\frac{1}{10} \sum_{i=10(k-1)+1}^{10 k} c_{i}(t)\right|_{t=1.0}$, where $k=1,2, \ldots, 800 / 10$. That is, we take every 10 nearby nodes in the ordering as a group. The $y$-coordinate represents the average value of the coupling strengths $\left.c_{i}(t)\right|_{t=1.0}$ in a group. (For interpretation of the references to colour in this figure legend, the reader is referred to the web version of this article.)
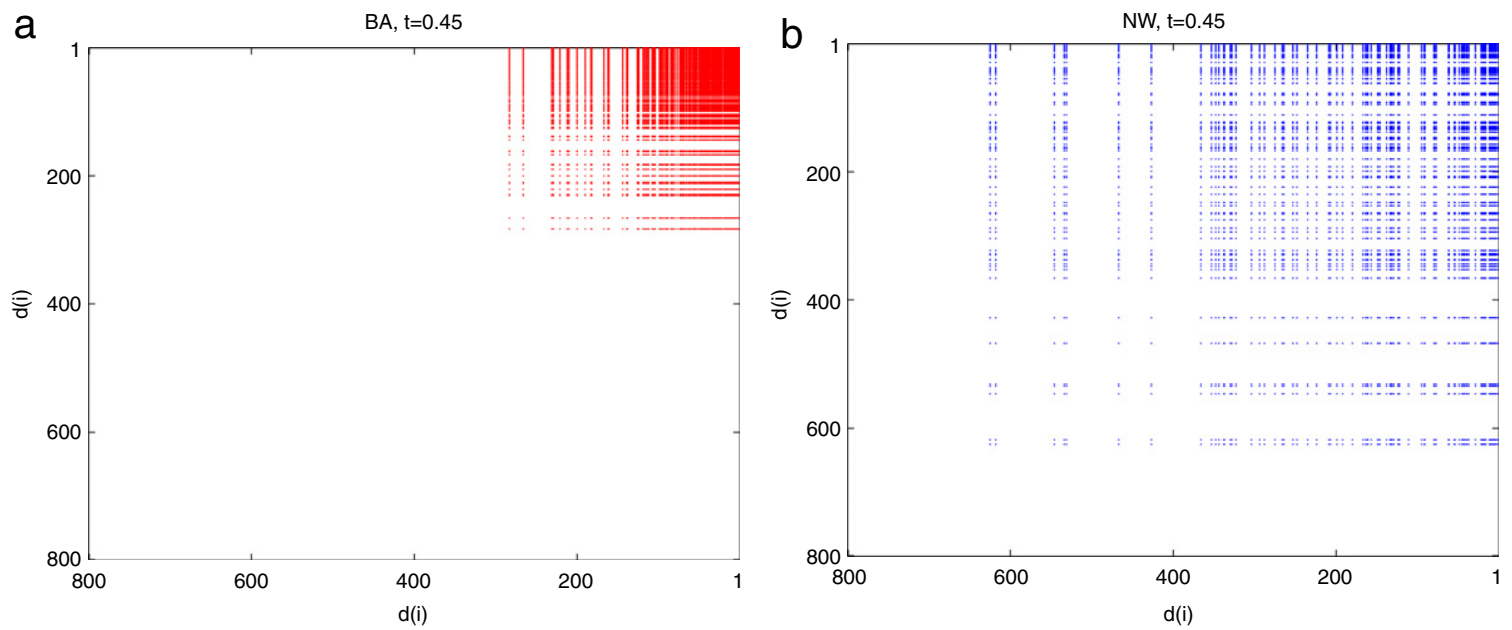

Fig. 7. The synchronization clusters in networks in the process of generalized synchronization at $t=0.45$, shown in the parameter space $(d(i), d(j))$. The threshold value of $\Delta_{t h r}$ equals 0.05 . A dot is plotted when $\varepsilon_{i j}=1$. Subfigure (a) is for the case of the scale-free (BA) network, and subfigure (b) is for the case of the small-world (NW) network, with $N=800$ nodes and $2388 \pm 2$ edges. Both of the two networks are with the same percentage of nodes in the GS states at $t=0.45$. However, the BA network shows self-organized hierarchical GS behavior while the NW network does not.

the size of cluster is smaller at time $t$. In simulations, the threshold value of $\Delta_{\text {thr }}$ is set to be 0.05 as in Section 4.2 , and we take $t=0.45$ in the GS processes of the above two simulations. The generalized synchronization clusters in the scale-free network and the small-world network are represented in Fig. 7(a) and (b), respectively. All nodes have been ranked in the ordering $d(1) \geq d(2) \geq \cdots \geq d(i) \geq \cdots \geq d(N)$. In Section 4.2, it has been shown in Fig. 3(b) that for both small-world and scale-free networks, there are about $20 \%$ of the nodes are in GS states at $t=0.45$. According to Fig. 7(a) of the case for the scale-free network, almost all the oscillators in GS states form a cluster (in a dense rectangle) with degrees $d(i)>d_{t h r}$, where $d_{t h r}$ is the threshold degree around $d(300)$. It shows a hierarchical structure which is determined by the connection degrees. However, in the small-world case, the threshold degree $d_{t h r}$ reaches $d_{620}$, and the rectangle in Fig. $7(\mathrm{~b})$ is much sparser than that in Fig. 7(a). One can conclude that scale-free networks form GS via self-organized clustering, while smallworld networks do not. In addition, it is suggested in Fig. 7(a) that nodes with more connections (hubs) are GS synchronized more closely and constitute the dynamical core of the hierarchical (scale-free) networks.

\subsection{Further investigation}

In order to further reveal how the heterogeneity of a network affects the process of generalized synchronization, in this subsection, we study the generalized synchronization for a family of networks generated by the model introduced in Ref. 
$\alpha=1.0$

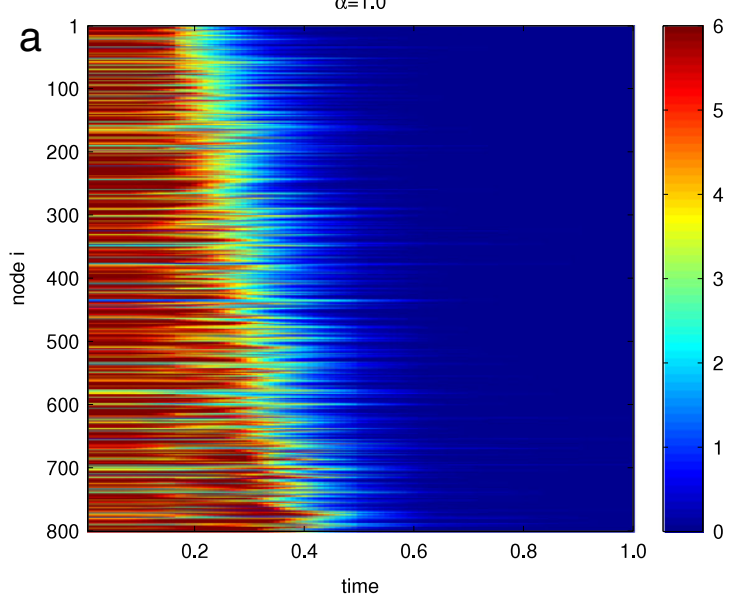

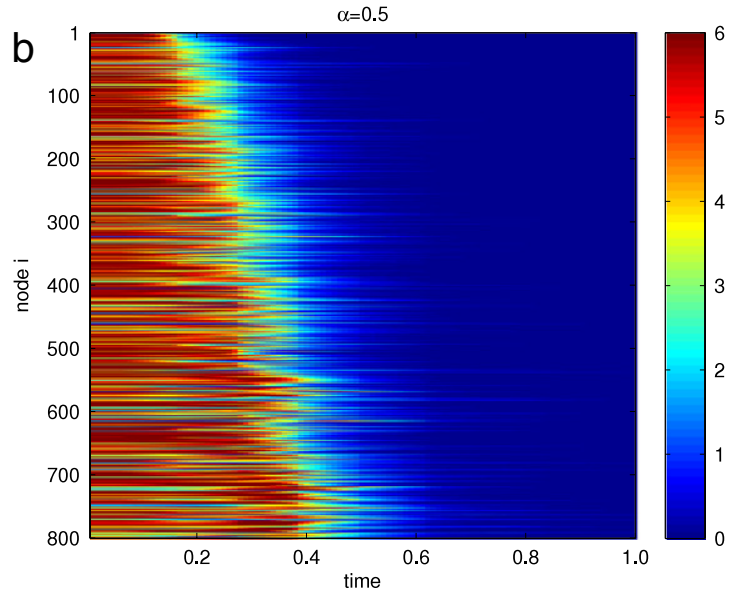

Fig. 8. Visualizations of the processes to global generalized synchronization via adaptive couplings for different network topologies parameterized by $\alpha$ [25]. $\alpha=0$ corresponds to the BA network, $\alpha=1$ the ER network, and $0<\alpha<1$ an interpolating network. In the simulations, each network is with $N=800$ nodes, and the average node degree $\langle k\rangle=6$. Subfigure (a) shows the case for $\alpha=1$, and subfigure (b) shows the case for $\alpha=0.5$. (For interpretation of the references to colour in this figure legend, the reader is referred to the web version of this article.)
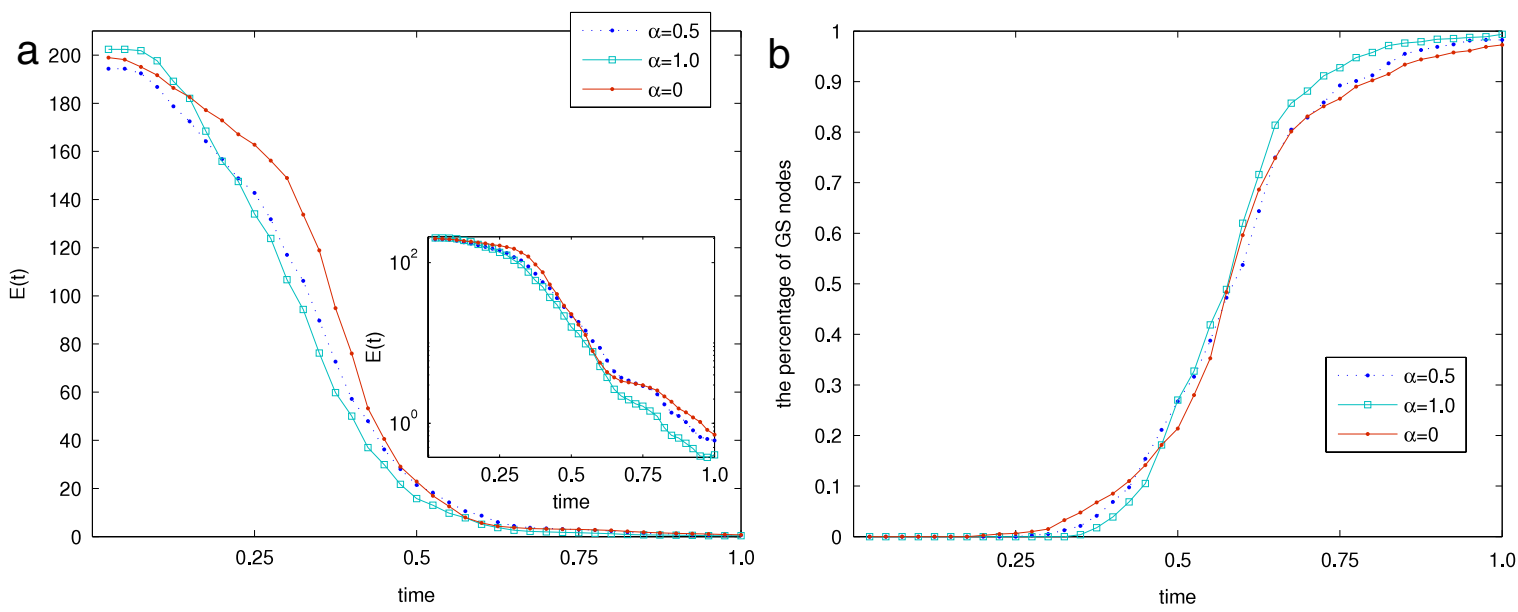

Fig. 9. Global generalized synchronization curves for different network topologies parameterized by $\alpha$ [25]. $\alpha=0$ corresponds to the BA network, $\alpha=1$ the ER network, and $\alpha=0.5$ an interpolating network. Each network is with $N=800$ nodes, and the average node degree $\langle k\rangle=6$. Fig. 9 (a) shows the evolutions of errors $E(t)=\sum_{i=1}^{N} E_{i}(t)$ for the three networks. For the sake of clarity, a smaller figure in subfigure (a) is drawn in linear-log scales. Subfigure (b) shows the percentages of the nodes that have achieved GS in the adaptive evolution processes.

[25] parameterized by $1-\alpha \in[0,1]$, which measures the degree of the structural heterogeneity of the generated network. The algorithm can be described as follows [25,26]: Assuming the final size of the network is $N$, the network is built up starting from a set of fully connected $m_{0}$ nodes and a set $U(0)$ of $N-m_{0}$ unconnected nodes. At each time step, a new node (having not been selected before) is chosen from the set $\mathcal{U}(0)$ and linked to $m$ other nodes. Each the $m$ links is attached in two ways: (a) with probability $\alpha$ the selected node is linked to a randomly chosen node from the whole set of the $N-1$ remaining nodes; (b) with probability $1-\alpha$ a link is established following a linear preferential attachment strategy [2]. After repeating this process for $N-m_{0}$ times, the network is generated and it interplays between the Erdös-Rényi (ER) random graph [3] (corresponding to $\alpha=1$ ) and the Barabási-Albert (BA) network (corresponding to $\alpha=0$ ). When $1-\alpha$ is near to 0 , it means that the network is homogeneous; when $1-\alpha$ is near to 1 , it means the network is heterogeneous. In the following simulations, we change the values of $\alpha$ to capture different behaviors in the process to achieve global GS.

We have performed extensive numerical simulations for each network for the time interval [0, 1.0] with step size $\delta t=0.025$. We also take the node's dynamics to be the three-dimensional classical Lorenz oscillators as shown in Eq. (6), where the parameters $\alpha_{i}$ are chosen to be different by the uniform randomization in the interval [28.0, 30.0].

Figs. 8 and 9 reveal different patterns of generalized synchronization emerging in a family of complex networks with topologies between homogeneous and heterogeneous ones. The color maps in Fig. 8(a) and (b) describe the visualizations of the processes to global GS for two complex networks with $\alpha=1.0$ and $\alpha=0.5$ respectively. As is known, when $\alpha=0.5$, it is an intermediate complex network between ER random network and scale-free (BA) network. Comparing Fig. 8(a) with 
Fig. 1(a), one can see that the process to achieve GS in ER random network $(\alpha=1.0)$ is quite different from the situation in the scale-free network $(\alpha=0)$. Most oscillators achieve GS states at approximately the same time. This is consistent with the fact that the ER random network has approximately homogeneous degree distribution, while the scale-free network is with huge hubs. In addition, a transition behavior in GS process in the intermediate network with $\alpha=0.5$ can be observed in Fig. 8(b), where the time interval for being in partial GS states $(0.2<t<0.8)$ is longer than that of the ER random network, and shorter than that of the scale-free network. The curves $E(t)$, defined in Section 4.2 , and the percentages of nodes in GS states at $t$, are shown in Fig. 9, for three network topologies with $\alpha=0, \alpha=0.5$ and $\alpha=1.0$. In Fig. 9(b), it shows that the partial GS first occurs in the scale-free network $(\alpha=0)$ at an early stage, but the global GS state is delayed later on. The situation is opposite in the model with $\alpha=1.0$. And an intermediate behavior in the process of non-GS $\rightarrow$ partial GS $\rightarrow$ global GS is shown in the network parameterized by an intermediate value $\alpha=0.5$. It can be concluded that, as the heterogeneity increases, the appearance of the partial GS is anticipated, but at the same time, the appearance of the global GS state is delayed. Similar phenomena, shown in Fig. 9, haven also been observed in Ref. [26] where complete synchronization is investigated and is different than our results for adaptively adjusting coupling strengths.

\section{Discussion: GS vs CS}

In this section, we compare the generalized synchronization with the complete synchronization in complex dynamical networks. For simplicity, only identical nodes and uniform coupling strengths are considered in the following analysis. Firstly, we briefly state an important result for the complete synchronization. The dynamics of a dynamical network of $N$ coupled oscillators is described by

$$
\dot{x}_{i}(t)=f\left(x_{i}(t)\right)-c \sum_{j=1}^{N} L_{i j} \Gamma x_{j}(t), \quad i=1,2, \ldots, N,
$$

where $x_{i}$ is the state of oscillator $i$ and $f\left(x_{i}(t)\right)$ governs the dynamics of each individual oscillator. $\Gamma$ is an inner coupling matrix. $L=\left(L_{i j}\right)$ is the Laplacian matrix of the underlying graph, where $L_{i j}=-1$ if there is a link between node $i$ and node $j$, and 0 otherwise; $L_{i i}=-\sum_{i \neq j} L_{i j}$. Suppose the completely synchronized state of Eq. (9) is $\left\{x_{i}(t)=s(t), \forall j \mid \dot{s}(t)=f(s(t))\right\}$. The local stability of this solution can be estimated following the approach of the master stability function [27]. Small perturbation of the synchronization state $s(t)$ can be governed by the linear variational equations

$$
\delta \dot{x}_{i}=D f(s) \delta x_{i}-c \sum_{j=1}^{N} \Gamma\left(\delta x_{j}\right), \quad i=1,2, \ldots, N,
$$

where $D f(s)$ is the Jacobian with respect to $s$. Eq. (10) can be diagonalized into $N$ decoupled blocks of the form

$$
\dot{\eta}_{i}=\left(D f(s)-c \lambda_{i} \Gamma\right) \eta_{i}, \quad i=1,2, \ldots, N,
$$

where $\eta_{i}$ is the eigenmode associated with eigenvalue $\lambda_{i}$ of the matrix $L$. Here $\lambda_{1}=0$, resulting from the invariant manifold, corresponds to the eigenmode parallel to the synchronization manifold, and the other $N-1$ eigenvalues $\lambda_{j}$ correspond to the eigenmodes transverse to the synchronization manifold [24].

From Eq. (11), the speed of node $i$ converging to the synchronization manifold is mainly determined by $\lambda_{i}$, once oscillators' dynamics $f(\cdot)$ are given. We rank the eigenvalues of the matrix $L$ in an ascending ordering

$$
0=\lambda_{1} \leq \lambda_{2} \leq \cdots \leq \lambda_{N}
$$

In Ref. [28], it has been pointed out that the large eigenvalues in the spectrum series (12) imply the existence of hubs in the network, and the gaps between consecutive eigenvalues indicate community structures in the network. And also from the analysis of Eq. (11), one can explain the hierarchical (clustering) synchronization [24] phenomenon in scale-free networks since there exist some relatively large eigenvalues in the series (12) for scale-free networks.

As far as generalized synchronization in complex dynamical networks is considered, one can obtain the linearization of the perturbation systems,

$$
\dot{e}_{i}=\left(D f\left(x_{i}\right)-c \sum_{j=1}^{N} a_{i j} \Gamma\right) e_{i}, \quad i=1,2, \ldots, N
$$

Since $k_{i}=\sum_{j=1}^{N} a_{i j}$, the above equation can be rewritten into

$$
\dot{e}_{i}=\left(D f\left(x_{i}\right)-c k_{i} \Gamma\right) e_{i}, \quad i=1,2, \ldots, N .
$$

Let the number of total edges in the network be $\mathfrak{E}$. Then $\sum_{i=1}^{N} k_{i}=2 \mathfrak{E}$. Denote the node-node distribution as $P\left(k_{i} \mid k\right)=$ $k_{i} / \sum_{j} k_{j}$, then Eq. (13) can be written as

$$
\dot{e}_{i}=\left(D f\left(x_{i}\right)-c 2 \mathfrak{E} P\left(k_{i} \mid k\right) \Gamma\right) e_{i}, \quad i=1,2, \ldots, N .
$$


From Eq. (13), the node degree $k_{i}$ determines the convergence speed of node $i$ to the generalized synchronization manifold $\left\{x_{i}(t)=\hat{x}_{i}(t), \forall i\right\}$, when oscillators' dynamics $f(\cdot)$ are given. Also from Eq. (14), it can be concluded that several properties (difference between the GS behaviors in small-world and scale-free networks) illustrated in details in the paper can be explained by the heterogeneity in the degree distributions. For example, GS starts from a small parts of hub nodes with larger degrees, and then spreads to others nodes with smaller degrees, as shown by the color maps in Figs. 1(a), 2(a), 8. Heterogeneous networks are easier to be synchronized at an early stage, but harder to achieve the global GS later on, as shown in Figs. 3 and 9.

It has been illustrated in the above analysis that generalized synchronization is different from complete synchronization. However, the generalized synchronization also shares some commonalities with the complete synchronization in comparison. For example, the evolution patterns of generalized synchronization in small-world and scale-free networks (shown in Fig. 3) are similar to the results obtained in paper [29]: scale-free networks outperform small-world networks in the beginning, but the trend is reverted at a later stage.

Hierarchical structure in scale-free networks has also been studied in Ref. [24], where only the complete synchronization properties are discussed. It also suggests that hubs having large degrees play the leading role in forming the dynamical core in the process of synchronization. However, there is some difference in the simulation results: the effective synchronization clusters in scale-free networks in Ref. [24] can be described by the relationship $i+j \leq J_{t h}$, where $J_{t h}$ is a constant threshold; while they can be described by the relationship $d(i) \geq d_{t h r}$ here when GS is considered.

\section{Conclusions}

In this paper, we have investigated the existence and process of generalized synchronization in three typical complex dynamical networks including scale-free networks, small-world networks, and a family of interpolating networks. The coupling strengths are adjusted adaptively based on nodes' local GS information. It has been demonstrated theoretically and numerically that for any given initial coupling strengths, generalized synchronization can take place in complex networks consisting of large nonidentical dynamical systems. It has also been found that, there exists a general process to global GS: non-GS $\rightarrow$ partial GS $\rightarrow$ global GS. In other words, not all nodes achieve GS state at the same time. As for the process of GS, it can be roughly stated that GS starts from a small part of hub nodes with larger degrees first, and then spreads to the other nodes with smaller degree. Furthermore, it has been shown that the processes of generalized synchronization in scale-free networks and small-world networks are different: there are hierarchical structures of the GS behavior in scalefree networks, but not in small-world networks. This can be explained by their distinct distributions of nodes' degrees. For small-world networks, the probability $p$ also affects the process of generalized synchronization. Finally, since adaptive coupling scheme is introduced, it has been illustrated that the coupling strengths become weighted differently partly as a result of the network topologies. In the future, we are interested in exploring more GS properties in more realistic networks; in particular, we want to look into different mechanisms to adaptively adjust the coupling strengths in different types of networks.

\section{Acknowledgements}

The authors would like to thank the anonymous reviewers for their comments. This work was supported by National Natural Science Foundation of China under Grants 70771084 and 60974081, by National Basic Research (973) Program of China under Grant 2007CB310805. The work of Liu and Cao was also supported in part, by grants from the Dutch Organization for Scientific Research (NWO) and the Dutch Technology Foundation (STW).

\section{References}

[1] D.J. Watts, S.H. Strogatz, Collective dynamics of 'small-world' networks, Nature 393 (6684) (1998) 440-442.

[2] A.L. Barabási, R. Albert, Emergence of scaling in random networks, Science 286 (5439) (1999) 509-512.

[3] P. Erdös, R. Rényi, On random graphs I, Publ. Math. Debrecen 6 (1959) 290-297.

[4] A. Arenas, A.D. Guilera, J. Kurths, et al., Synchronization in complex networks, Phys. Rep. 469 (2008) 93-153.

[5] J. Lü, X. Yu, G. Chen, Chaos synchronization of general complex dynamical networks, Physica A 334 (1-2) (2004) 281-302.

[6] J. Lü, X. Yu, G. Chen, D. Cheng, Characterizing the synchronizability of small-world dynamical networks, IEEE Trans. Circuits Syst. I 51 (2004) 787-796.

[7] C.W. Wu, L.O. Chua, Synchronization in an array of linearly coupled dynamical systems, IEEE Trans. Circuits Syst. I, Reg. Papers 42 (8) (1995) 430-447.

[8] N.F. Rulkov, M.M. Sushchik, L.S. Tsimring, H.D.L. Abarbanel, Generalized synchronization of chaos in directionally coupled chaotic systems, Phys. Rev. E 51 (2) (1995) 980-994.

[9] H. Suetani, Y. Iba, K. Aihara, Detecting generalized synchronization between chaotic signals: A kernel-based approach, Physics A 39 (34) (2006) 10723-10742.

[10] H.D.I. Abarbanel, N.F. Rulkov, M.M. Sushchik, Generalized synchronization of chaos: The auxiliary system approach, Phys. Rev. E 53 (5) (1996) $4528-4535$.

[11] X. Wu, W.X. Zheng, J. Zhou, Generalized outer synchronization between complex dynamical networks, Chaos 19 (013109) (2009).

[12] I. Grosu, E. Padmanaban, P.K. Roy, S.K. Dana, Designing coupling for synchronization and amplification of chaos, Phys. Rev. Lett. 100 (234102) (2008).

[13] G.J. Peng, Y.L. Jiang, F. Chen, Generalized projective synchronization of fractional order chaotic systems, Physica A 387 (14) (2008) $3738-3746$.

[14] Y.C. Hung, Y.T. Huang, M.C. Ho, C.K. Hu, Paths to globally generalized synchronization in scale-free networks, Phys. Rev. E 77 (016202) (2008).

[15] S.G. Guan, X.G. Wang, X.F. Gong, K. Li, C.H. Lai, The development of generalized synchronization on complex networks, Chaos 19 (013130) (2009).

[16] X. Xu, Z. Chen, G. Si, X. Hu, P. Luo, A novel definition of generalized synchronization on networks and a numerical simulation example, Comput. Math. Appl. 56 (11) (2008) 2789-2794. 
[17] F. Sorrentino, E. Ott, Adaptive synchronization of dynamics on evolving complex networks, Phys. Rev. Lett. 100 (114101) (2008).

[18] J. Lü, G. Chen, A time-varying complex dynamical network model and its controlled synchronization criteria, IEEE Trans. Automat. Control 50 (6) (2005) 841-846.

[19] P. Li, Z. Yi, Synchronization analysis of delayed complex networks with time-varying couplings, Physica A 387 (2008) $3729-3737$.

[20] C. Zhou, J. Kurths, Dynamical weights and enhanced synchronization in adaptive complex networks, Phys. Rev. Lett. 96 (164102) (2006).

[21] W. Lu, Adaptive dynamical networks via neighborhood information: Synchronization and pinning control, Chaos 17 (023122) (2007).

[22] A. Barrat, M. Barthélemy, A. Wespignani, Modelling the evolution of weighted networks, Phys. Rev. E 70 (066149) (2004).

[23] M.E.J. Newman, D.J. Watts, Renormalization group analysis of the small-world network model, Phys. Lett. A 263 (4-6) (1999) 341-346.

[24] C. Zhou, J. Kurths, Hierarchical synchronization in complex networks with heterogenous degrees, Chaos 16 (015104)(2006).

[25] J. Gómez-Gardeñes, Y. Moreno, From scale-free to Erdos-Réyi networks, Phys. Rev. E 73 (056124) (2006).

[26] J. Gómez-Gardeñes, Y. Moreno, A. Arenas, Synchronizability determined by coupling strengths and topology on complex networks, Phys. Rev. E 75 (066106) (2007).

[27] L.M. Pecora, T.L. Carroll, Master stability functions for synchronized coupled systems, Phys. Rev. Lett. 80 (1998) 2109-2112.

[28] A. Arenas, A.D. Guilera, C.J.P. Vicente, Synchronization reveals topological scales in complex networks, Phys. Rev. Lett. 96 (114102) (2006).

[29] J. Gómez-Gardeñes, Y. Moreno, A. Arenas, Paths to synchronization on complex networks, Phys. Rev. Lett. 98 (034101) (2007). 